\title{
Utility of contrast-enhanced echocardiography in the diagnosis and follow-up of a pseudoaneurysm of the aortic arch
}

\author{
$\mathrm{Yu} \mathrm{Wu}{ }^{1}$, Yuman $\mathrm{Li}^{2}$, Jia $\mathrm{Xu}^{3}$, Wang Menghe ${ }^{4}$, and Qing $\mathrm{Lv}^{5}$ \\ ${ }^{1}$ Department of Ultrasound, Union Hospital, Tongji Medical College, Huazhong University \\ of Science and Technology, Wuhan, 430022, China \\ ${ }^{2}$ Union Hospital, Tongji Medical College, Huazhong University of Science and Technology, \\ ${ }^{3}$ Huazhong University of Science and Technology Tongji Medical College \\ ${ }^{4}$ Affiliation not available \\ ${ }^{5}$ Union Hospital, Tongji Medical College, Huazhong University of Science and Technology
}

July 22, 2021

\begin{abstract}
Spontaneous pseudoaneurysm of the aortic arch is an exceptionally rare and potentially life-threatening condition.Diagnosis of pseudoaneurysms of the aortic arch mainly depends on imaging examinations.Contrast-enhanced echocardiography, as a non-invasive, bed-side and radiation-free tool, plays an important role in the rapid diagnosis and postoperative follow-up of pseudoaneurysms of the aortic arch.
\end{abstract}

Utility of contrast-enhanced echocardiography in the diagnosis and follow-up of a pseudoaneurysm of the aortic arch

$\mathrm{Yu} \mathrm{Wu}^{1, *}, \mathrm{MD}, \mathrm{PhD}$, Yuman $\mathrm{Li}^{1, *}, \mathrm{MD}, \mathrm{PhD}, \mathrm{Jia} \mathrm{Xu}^{1, *}$, Menghe Wang ${ }^{2}, \mathrm{MM}$, and Qing $\mathrm{Lv}^{1}, \mathrm{MD}, \mathrm{PhD}$

1 Department of Ultrasound, Union Hospital, Tongji Medical College, Huazhong

University of Science and Technology, Wuhan, 430022, China.

2 The First Affiliated Hospital of Zhengzhou University, Zhengzhou, 450000, China

*Contributed equally

Corresponding author:

Qing Lv, MD, PhD.

Tel: 86-27-85726430; Fax: 86-27-85726386.

E-mail address: lvqing1987@hust.edu.cn

Department of Ultrasound, Union Hospital, Tongji Medical College, Huazhong

University of Science and Technology, 1277 jiefang avenue, Wuhan, 430022, China

A 47-year-old female presented to our hospital with hoarseness for two years. She had no history of surgery, trauma, hypertension, collagen disease or other potential infectious diseases. Transthoracic echocardiography revealed a mass adjacent to the lesser curvature of aortic arch (Figure 1A). Color flow Doppler imaging did not clearly show if the mass communicated with the aortic arch. Subsequently, two-dimensional and three-dimensional contrast-enhanced echocardiography clearly demonstrated that a large pseudoaneurysm 
$(5.5 \times 6.5 \mathrm{~cm})$ communicated with the aortic arch via an $8-\mathrm{mm}$ wide defect located on the opposite side of the left subclavian artery (Figures $1 \mathrm{~B}$ and 1C, Videos 1 and 2). A thrombus within the pseudoaneurysm cavity was also observed. Computed tomography (CT) confirmed the diagnosis of pseudoaneurysm of the aortic arch (Figure 1D). The patient underwent a successful pseudoaneurysm resection and the aorta replacement. Postoperative two-dimensional and three-dimensional contrast-enhanced echocardiography showed no leakage around the vascular graft (Figures $1 \mathrm{E}$ and $1 \mathrm{~F}$, videos 3 and 4). 23 months later, follow-up twodimensional echocardiography and contrast-enhanced echocardiography showed a recurrent pseudoaneurysm $(3.0 \times 1.7 \mathrm{~cm})$ arising from the proximal part of descending aorta (Figures $2 \mathrm{~A}$ and B, video 5$)$. CT angiography revealed the recurrent pseudoaneurysm originating from the proximal end of descending aorta (Figure $2 \mathrm{C})$. Unfortunately, the patient declined any form of treatment. At the half-month follow-up, the patient died of a ruptured pseudoaneurysm.

Spontaneous pseudoaneurysm of the aortic arch is an exceptionally rare and potentially life-threatening condition. Diagnosis of pseudoaneurysms of the aortic arch mainly depends on imaging examinations. Contrastenhanced echocardiography, as a non-invasive, bed-side and radiation-free tool, can greatly improve visualization of the pseudoaneurysm [1]. In this case, the extension, neck and mural thrombus of the pseudoaneurysm are well visualized after contrast administration. Our case highlights the value of contrast-enhanced echocardiography in the rapid diagnosis and postoperative follow-up of pseudoaneurysms of the aortic arch.

Informed consent: Written informed consent was given by the patient's relatives.

\section{References}

1. Sehmi JS, West C, Khattar R, Senior R, Chahal NS. Mass confusion: defining aortic pathology with ultrasound contrast. Circulation 2015; 132: 1433-1434.

Figure legend:

Figure 1. Preoperative and postoperative imaging. (a) Echocardiography showing a mass (asterisk) adjacent to the lesser curvature of aortic arch. (b-c) Two-dimensional and three-dimensional contrast-enhanced echocardiography displaying a pseudoaneurysm communicating with the aortic arch (arrow). (d) CT angiography confirming the presence of pseudoaneurysm of the aortic arch. (e-f) Postoperative two-dimensional and threedimensional contrast-enhanced demonstrating no leakage around the vascular graft. AAO: Ascending aorta; DAO: Descending aorta.

Figure 2. Follow-up imaging (23 months after surgery). (a) Echocardiography revealing a recurrent pseudoaneurysm (asterisk) arising from the proximal part of the descending aorta. (b) Contrast-enhanced echocardiography demonstrating a recurrent pseudoaneurysm of the descending aorta. (c) CT angiography reconstruction indicating the recurrent pseudoaneurysm originating from the proximal descending aorta.

Video legend:

Video 1: Preoperative two-dimensional contrast-enhanced echocardiography displaying a pseudoaneurysm communicating with the aortic arch.

Video 2: Preoperative three-dimensional contrast-enhanced echocardiography revealing a pseudoaneurysm communicating with the aortic arch.

Video 3: Postoperative two-dimensional contrast-enhanced demonstrating no leakage around the vascular graft.

Video 4: Postoperative three-dimensional contrast-enhanced showing no leakage around the vascular graft.

Video 5: Follow-up contrast-enhanced echocardiography demonstrating a recurrent pseudoaneurysm of the descending aorta. 

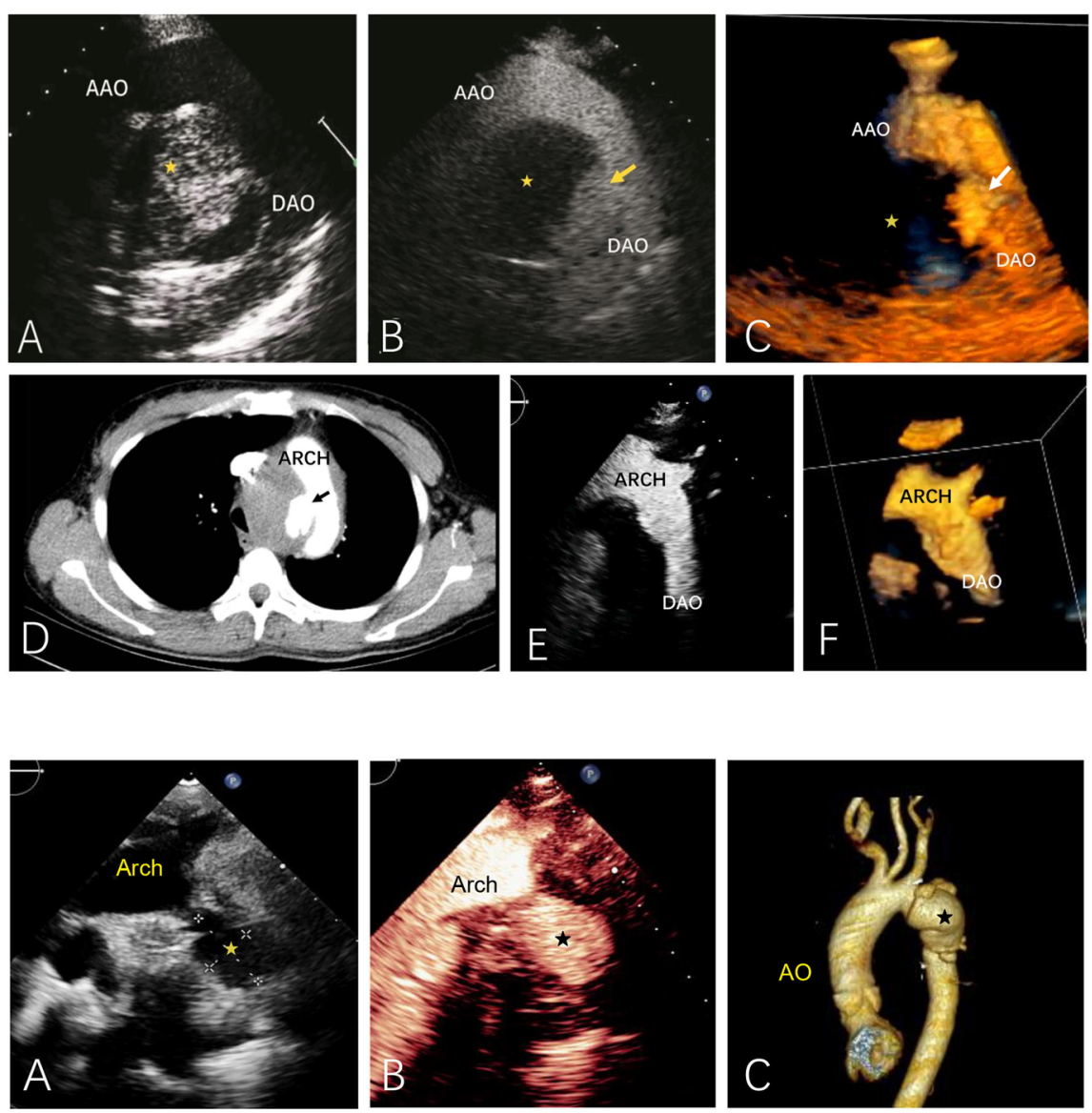